\title{
Management of Chemotherapy Induced Neutropenia - an Unmet Clinical Need
}

\author{
Ananya Gupta ${ }^{1 *}$, Benazir Abbasi ${ }^{2}$ and Sanjeev Gupta ${ }^{2}$ \\ ${ }^{1}$ Department of Physiology, National university of Ireland Galway, Ireland \\ ${ }^{2}$ Department of Pathology, National university of Ireland Galway, Ireland
}

*Corresponding author: Ananya Gupta, Discipline of Physiology, School of Medicine, National university of Ireland Galway, Ireland.

To Cite This Article: Ananya Gupta. Management of Chemotherapy Induced Neutropenia - an Unmet Clinical Need. Am J Biomed Sci \& Res. 2019 - 4(5). AJBSR.MS.ID.000823. DOI: 10.34297/AJBSR.2019.04.000823

Received: July 27, 2019 | Published: August 14, 2019

\begin{abstract}
One of the major side effects of cancer chemotherapy is myelosuppression leading to an obligate period of neutropenia. Systemic infection resulting in neutropenic sepsis is the leading cause of morbidity and mortality in cancer patients. Due to these reasons chemotherapy induced neutropenia has been considered as an "oncologic emergency". The current system of management of febrile neutropenia involves administration of prophylactic antimicrobials and hematopoietic growth factor supplements (granulocyte colony stimulating factor G--CSF). However, neutropenia is detected in these patients only after the onset of infection limiting the efficacy of these therapeutic interventions. Repeated infections results in prolonged periods of hospitalisation, delay in treatment and chemotherapy dose reductions. Therefore, timely detection of neutrophil nadir and prevention of systemic infection can significantly improve the outcome of cancer treatment.

Keywords: Cancer; Chemotherapy; Neutropenia; fever; Sepsis; Growth factors; Antibiotics; Oncologic emergency

Abbreviation: CIN: Chemotherapy Induced Neutropenia; FN: Febrile Neutropenia; NICE: National Institute for Health and Care Excellence; CBC: Complete Blood Cell Count; ANC: Absolute Neutrophil Count; G-CSF: Granulocyte Colony Stimulating Factor; RCT: Randomized Control Trial; EZMO: European Society for Medical Oncology; MASCC: Multinational Association of Supportive Care of Cancer; EORTC: European Organization for Research and Treatment of Cancer; NCEPOD: National Confidential Enquiry into Patient Outcome and Death
\end{abstract}

\section{Introduction}

Chemotherapy treatments for cancer are well--known to be associated with a depletion of white blood cells, in particular neutrophils, causing a condition known as chemotherapy--induced neutropenia (CIN), as described in Figure 1. Where neutropenia is significant, it is associated with an increased risk of systemic infections that lead to sepsis and death $(1,2,3,7,9)$. As per the National Institute for Health and Care Excellence (NICE) guidelines, cancer centres instruct patients receiving chemotherapy to "take their temperature if they feel unwell and, and to report this to the centre as soon as possible". Patients having fever or febrile neutropenia (FN) then attend hospital and undergo assessment including a complete blood cell count (CBC). Fever in FN indicates an ongoing infection and is considered an oncologic emergency $(7,9)$. The CBC requires admission to hospital for access to gold standard equipment and hospital personnel to complete the blood draw and subsequent analysis. If a blood test reveals that neutrophils are low, the patient is admitted to hospital for futher treatment with intra-venous antibiotics, growth factors and blood transfusions.
The risk increases with the duration and severity of the neutropenia. If neutropenia is detected early, a number of prevention methods can be used to lower the risk of infection and prevent FN; namely the use of granulocyte colony--stimulating factors (G-CSF), which stimulates production of new neutrophils and the use of prophylactic antibiotics. However, a meta--analysis has shown that overall mortality is not influenced significantly by the use of antibiotics or growth factors $(4,6)$. The current treatment regime provides only a small reduction in infection--related mortality. The critical element in reducing neutropenia related mortality is early diagnosis before the onset of infection and fever resulting in reduced need for hospitalisation and a faster neutrophil recovery.

\section{Chemotherapy Induced Neutropenia}

Neutropenia is a serious adverse effect frequently associated with cancer chemotherapy $(1,7)$. Loss of neutrophils causes disruption of immune defense mechanisms and increases the likelihood for infections. Infections lead to fever known as febrile neutropenia (FN). According to the European Society for Medical 
Oncology (ESMO), FN is defined by oral temperature greater than $38.5{ }^{\circ} \mathrm{C}$ for two consecutive readings within $2 \mathrm{~h}$ and an absolute neutrophil count (ANC) below $0.5 \times 109 /$ (1). Neutrophils (Figure 1) constitute the first line of defense against invading pathogens.
Reduction of ANC leads to a predisposition to infection caused by bacteria and fungi $[1,2]$. Chemotherapy induced Neutropenia (CIN) and its consequences are described in (Figure 1).

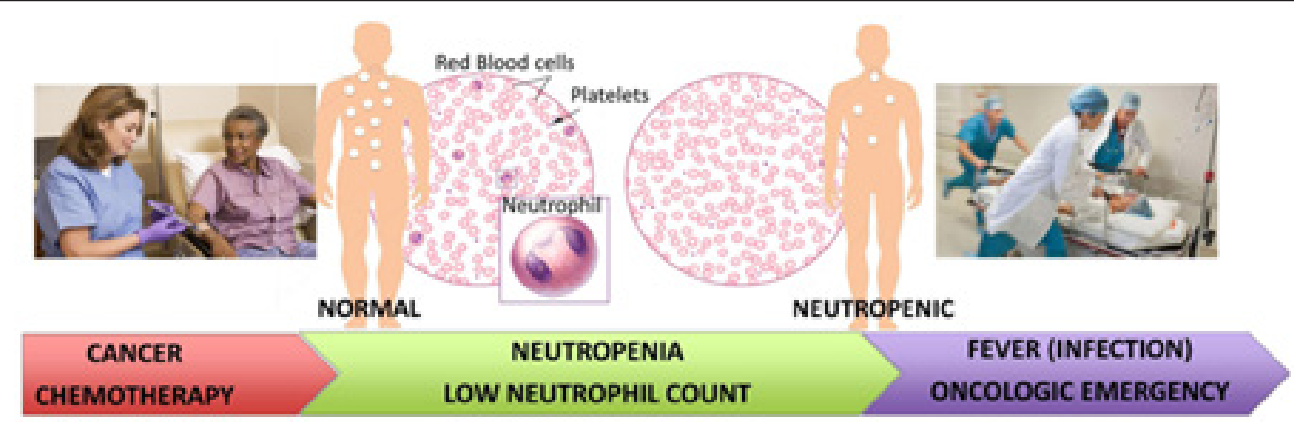

Figure 1: Current practice in management of CIN in cancer patients. Schematic representation of the onset of chemotherapy induced neutropenia and its consequences. A rapid response within 2 hours of onset of fever is crucial to prevent sepsis and death.

Timely diagnosis of neutropenia before the actual onset of infection provides the opportunity for appropriate clinical intervention (with G--CSF and antibiotics). The biggest challenge in the diagnosis of FN is patients recognizing the symptoms [1,3]. On referral to hospital, rapid treatment of FN patients with broad-spectrum antibiotics is paramount to prevent serious infections. Neutrophil count is currently diagnosed hospital admission using an automated cell counter. This results in a fatal delay before treatment can begin. Early detection of the onset of neutropenia can improve diagnosis and management. FN is an indication of underlying infection and must be considered as a "medical emergency" that requires immediate hospitalization for further evaluation and administration of hematopoietic growth factors and broad--spectrum antibiotics $[1,4,5]$. Neutropenia has a negative impact on the delivery of planned chemotherapy regimes, with dose reductions and treatment delays that compromise long--term clinical outcomes and cancer treatment [3]. There is no monitoring system available at present to detect neutropenia at the time of onset in an at--home or near--patient setting.

\section{Incidence, management strategies and impact}

The incidence of FN can be as high as 50\% in solid tumors and $\geq 80 \%$ in hematological malignancies [4,5]. Globally, the mortality rates associated with $\mathrm{FN}$ amongst cancer patients is extremely high ranging from 10--21\% [4]. Chemotherapy--induced neutropenia is the major cause for infection--related complications in cancer patients [4], including fungal infections, sepsis resulting from severe bacterimia, pneumonia and other respiratory tract infections, cerebrovascular disease and disorders of the liver and kidney [6,7]. Globally, the mortality rates associated with FN amongst cancer patients is extremely high ranging from 10 to $21 \%$ [8]. The risk of death is further increased due to the presence of co--morbidities in the patients which poses an additional risk, these include patient specific characteristics such as age, type of malignancy, treatment regime, performance status, health and nutritional status and infectious complications (Figure 2) [6,9].
Chemotherapy Induced Neutropenia: Risks, Symptoms and Complications

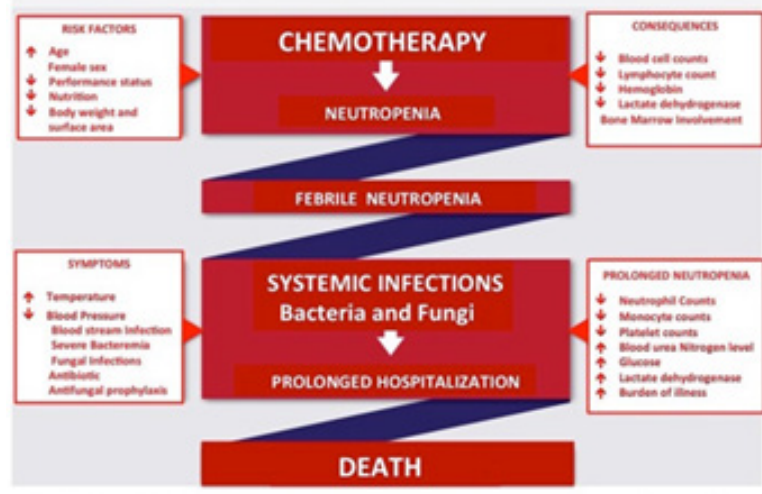

Figure 2: Schematic representation of the onset neutropenia and its complications.

In the current management if CIN occurs in the first few cycles of chemotherapy it results in significant dose reduction and delay in delivery of subsequent cycles, compromising effectiveness of treatment and disease--free/overall survival in patients treated with a curative intent $[3,10,11]$. Increasing age is associated with a higher risk of cancer. In addition age also leads to reduced bone marrow reserve and compromised lung, kidney and liver functions, further increasing the risk of chemotherapy--induced complications $[3,11]$. Other variables contributing to higher risk of developing neutropenia include the treatment regime, the chemotherapy drug or combination of drugs and associated toxicity (Figure 2) [1,2].

CIN when accompanied with fever needs to be treated immediately as the body can no longer defend itself against an active ongoing infection, which may result in systemic infection and sepsis $[3,11]$. Patients are required to be admitted to the hospital for further evaluation and treatment with broad--spectrum antibiotics must be initiated within hours of developing fever. The patient is monitored in the hospital until the neutrophil counts fully recover. They may receive therapeutic injections of myeloid growth factors (e.g., granulocyte colony--stimulating factor (G--CSF) to 
boost neutrophil production [2,5]. Duration of hospitalization varies from a few days to weeks depending on the duration and depth of neutropenia [12]. FN patients can be discharged from the hospital when their neutrophil count is greater than 0.5X109/1 and no evidence of infection has been found [12-14]. In many patients, particularly with age greater than 60 , and patients with hematological malignancies the recovery of neutrophils are very slow making prolonged hospitalization and multiple blood transfusions necessary [14].

In addition patients suffering from febrile neutropenia may be given prophylactic G--CSF during the next and subsequent chemotherapy cycles. Prophylactic G--CSF can significantly reduce the depth and duration of neutropenic nadir $[15,16]$. The long--acting peg--filgrastim has several advantages including convenience of administration to patient, improved compliance and greater potency [7]. Multiple randomized controlled trials (RCTs) have demonstrated the safety and efficacy of CSF use in patients admitted to the hospital with FN following cancer chemotherapy $[15,16]$. However, a recent review of FN patients who received G-CSF in addition to antibiotics showed no significant effect on overall mortality $[15,17]$. Though the duration and depth of neutropenia seemed to be reduced in participants receiving G--CSF and antibiotics compared to patients who received antibiotics alone, G--CSF did not significantly reduce the risk of infections and related complications from occurring during the neutropenic episode $[3,15]$. The risk of systemic infections and sepsis increases in direct proportion to the severity and duration of neutropenia [3]. Another study revealed that although myeloid growth factors, such as G-CSF reduce the duration and depth of neutropenia (Figure 3) but did not reduce infection and associated morbidity and mortality $[6,11,13]$.

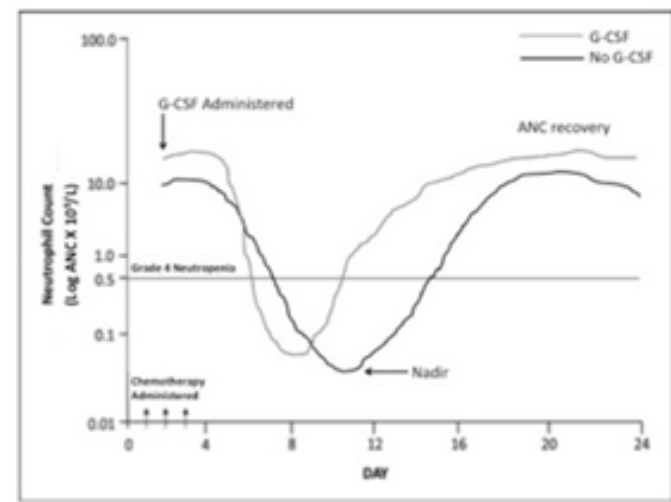

Figure 3: Neutropenic nadir duration and depth depends on therapy, dose, route of treatment and patient status. ANC recovery depends on regimen and patient status. G-CSF reduces neutrophil nadir duration and intensity.

The Multinational Association of Supportive Care of Cancer (MASCC) risk--index scoring system was designed for identifying cancer patients with high risk of CIN [18]. The tool consists of 8 factors that can be easily assessed upon admission with FN.
Patients with a score greater than 21 are considered low risk, and patients with lower scores are considered at a higher risk and need more intensive management. Poor predictors of prognosis include age greater than 60 , moderate symptoms with febrile neutropenia, hypotension, dehydration, inpatient status, and prior fungal infection [18]. The MASCC Risk Index can identify patients at low risk for complications and that it could be used to select patients for further monitoring and administering cost--effective therapies. The MASCC Risk--Index Scoring system is shown in (Table 1). This tool was endorsed in 2002 by the Infectious Diseases Society of America and has also been adopted by the European Society of Medical Oncology [16]. The MASCC risk scoring system is extremely useful as it allows health care providers to determine a patient's risk for developing medical complications from CIN and infections. A patient's treatment plan can be determined based on their risk category. It is important to know whether a patient is expected to be at high risk for developing FN or has a history of FN, in order to take appropriate prophylactic measures [19]. The detailed treatment plan and clinical management of CIN is described in $[5,19]$.

\begin{tabular}{|c|c|}
\hline \multicolumn{2}{|l|}{ Table 1: MASCC Risk index and weights. } \\
\hline Characteristics & Weight \\
\hline Burden of febrile neutropenia with or no mild symptoms1 & 5 \\
\hline No Hypotension (systolic blood pressure > 90 mmof Hg) & 5 \\
\hline No chronic obstructive pulmonary disease & 4 \\
\hline $\begin{array}{c}\text { Solid tumor orhematological malignancy with no previous } \\
\text { fungal infection }\end{array}$ & 4 \\
\hline No dehydration requiring parenteral fluids & 3 \\
\hline Burden of neutropenia with moderate symptoms & 3 \\
\hline Outpatient status & 3 \\
\hline Age $<60$ & 2 \\
\hline
\end{tabular}

Burden of febrile neutropenia refers to general clinical status under the influence of the neutropenic episode. Score no or mild symptom --5 , moderate symptom --3 , severe symptom --0 , moribund --0.

Maximum score possible is 26. A score of $>21$ is considered low risk and a score of $<21$ is considered high risk. (Source: http:// www.mascc.org/mascc--fn--risk--index--score)

The European Organization for Research and Treatment of Cancer (EORTC), recommends routine prophylactic use of G--CSF in those receiving a regimen with a FN risk score of greater than $20 \%$ in order to maintain effective chemotherapy dose intensity $[[1,2,4]$ (Figure 4). For patients with a score of risk score of 10 to $20 \%$ an individual assessment needs to be done to decide on G-CSF administration. This intermediate category represents $52.5 \%$ of cancer patients and there is significant variability in treatment received [20]. Many patients belonging to this category often experience FN during their first cycle of chemotherapy. They are then classified as high risk and given G--CSF in subsequent cycles [20]. More research is therefore needed in the intermediate regimes 
to identify patients who are at risk of FN. The EORTC does not recommend use of G--CSF when the risk is less than 10\% (Figure 5). However, in patients where a dose intense chemotherapeutic regime is believed to be beneficial prophylactic G--CSF is deemed necessary in order to have survival benefit. G--CSF is also advised when dose intense chemotherapy is deemed essential for effective cancer treatment [1,4]. Timely detection and diagnosis of CIN would also therefore make it possible to administer timely and appropriate G-- CSF prophylaxis.

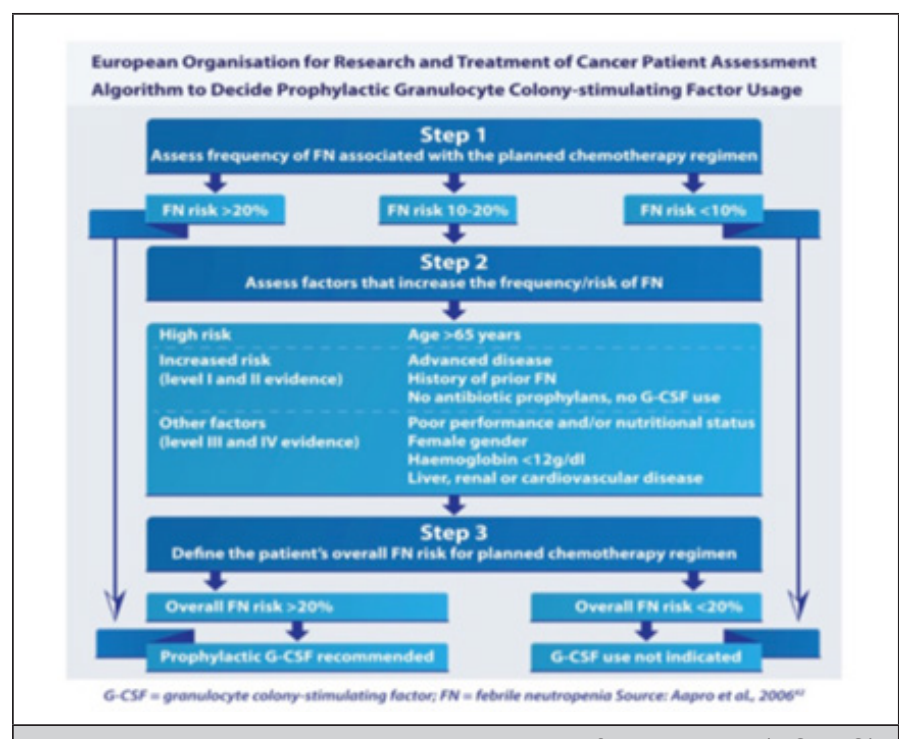

Figure 4: Risk models and management of neutropenia (EORTC).

The mortality and comorbidities associated with FN require immediate hospitalization and treatment with antimicrobial agents [10]. A recent study evaluating the resource use and cost of hospitalization for FN, within the Irish Healthcare system, found that the mean cost per FN episode was €8915 \pm 718 [21]. A study carried out by the Centers for Disease Control and Prevention, USA found that in 2012 there were 108,419 (91,560 adults and 16,859 children) cancer patients admitted to the hospital with CIN at a total cost of $\$ 2.7$ bn [22]. The mean length of stay for adults and children was 9.6 and 8.5 days respectively with a mean hospital cost of $\$ 24,770$ and $\$ 26,000$ per stay [22]. Furthermore, FN frequently compromises the chemotherapeutic treatment by requiring a dose reduction and/or delay of treatment cycles, thereby affecting treatment outcome [13]. Hence, management of CIN remains to be a yet unmet clinical need in the delivery of cancer chemotherapy.

A survey conducted by the National Confidential Enquiry into Patient Outcome and Death (NCEPOD) in 2008, titled "Systemic Anti Cancer Therapy: for better or for Worse?" reviewing the care received by patients who died within 30 days of delivery of chemotherapy, identified several serious shortcomings in the management of CIN in the existing practice [23]. Serious deficiencies were identified in education and awareness of the risks of FN in both patient and health--care professional. Also clear protocols for patient assessment, treatment and hospital admission were not available or properly implemented. The NCEPOD recommended that effective strategies for diagnosing and managing febrile neutropenia should be essential components of all cancer care services. A recent report on projected economic burden and loss of productivity associated with cancer related mortalities estimated approximately 233,000 projected deaths from all invasive cancers occurring in Ireland between 2011 and 2030 [18]. This will result in a lost of productivity valued at $€ 73$ billion [18]. These losses represent approximately $1.4 \%$ of Ireland's GDP annually. An annual $1 \%$ reduction in mortality reduces productivity losses due to all invasive cancers by $€ 8.5$ billion over 20 years [18]. Though the article did not discuss specifically the burden imposed by CIN however the potential impact of approximately $20 \%$ cancer related deaths occurring due to CIN is obvious. A recent study evaluated and estimated the cost and burden of hospitalization with FN within the Irish Healthcare system and found that average cost per FN episode was $€ 8915 \pm 718$ [21]. Of this the major cost was the hospital stay at $€ 6851 \pm 549$, and additional costs include antibiotics $€ 760 \pm 156$ laboratory tests $€ 538 \pm 47$ and blood transfusions $€ 525 \pm 189$ [21]. The study did not include the cost of G--CSF given to the patients. The mean length of stay was found to be $7.3 \pm 0.5$ days. There were 42 FN related hospital admissions at the University Teaching Hospital, Dublin reported in this study during one year (August 2008 - July 2009) [21]. This clearly outlines the incidence and impact of FN and the significant burden on the Healthcare system. Therefore, there is an immediate and urgent clinical need for rapid detection of neutropenia and prevention of sepsis to improve patient survival and outcome of cancer treatment.

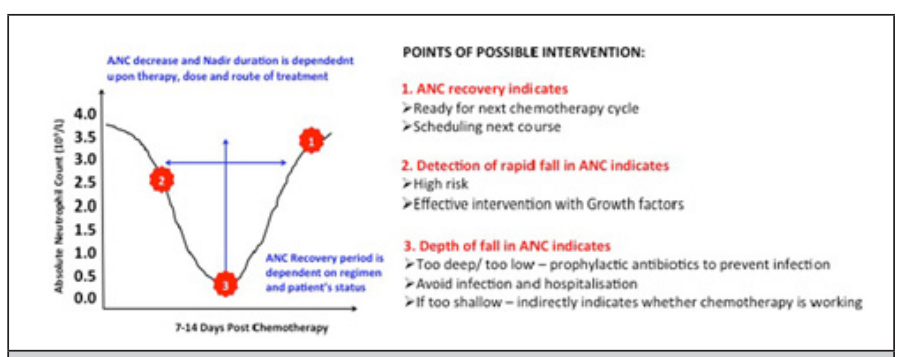

Figure 5: Monitoring Absolute Neutrophil count (ANC) leading to timely diagnosis and better management of CIN. 1,2,3 indicates the possible intervention points where monitoring of ANC can me of advantage in patient management. ANC monitoring to lead to timely detection and prevention of CIN avoiding hospitalization and treatment interruption. ANC monitoring could also help identifying the patients who could benefit from G-CSF administrated. ANC can also help to determine dose and time of next chemotherapy administrated.

\section{Conclusions}

One major obstacle faced in the management of FN is the timely diagnosis of neutropenia before the actual onset of infection. Timely diagnosis would provide a critical window of opportunity when appropriate clinical intervention (with G--CSF and antibiotics) can be made. The number one challenge in the diagnosis of febrile neutropenia is the patients ability to identify the signs and symptoms of CIN and realize that they are seriously ill - and the need to report immediately to the appropriate cancer care or hospital ward [3]. It is essential to educate all patients with cancer and receiving 
chemotherapy about the risk of FN, prior to beginning treatment. The second challenge in the management of febrile neutropenia is to make sure the patient's symptoms are properly documented and communicated so that they enter the appropriate care pathway as per local guidelines [3]. Any chemotherapy patient who has a fever associated with neutropenia should be regarded as a "medical emergency". It is essential for primary care givers to be aware of the patient's chemotherapy treatment and must immediately refer to the hospital.

On referral to hospital, all patients with febrile neutropenia must be treated rapidly to prevent serious infections and sepsis. So it is obvious that appropriate monitoring and early detection of when absolute neutrophil count (ANC) drops below $<0.5 \mathrm{x}$ $109 /$ indicating severe neutropenia and risk of infections will be extremely beneficial to the management of CIN. Patients can be alerted and themselves contact the appropriate hospital care for further evaluation and treatment. This will give valuable time when intervention with prophylactic G--CSF and/or antibiotic can prevent life--threatening infections from setting in. In addition patients diagnosed with neutropenia could be given ex--vivo expanded neutrophils to prevent onset of serious infections as well as to rapidly bring any ongoing infections under control, giving patient the chance to recover fast and continue on their treatment regimen.

The current clinical need can be addressed by introducing a regular system of monitoring blood cell counts in patients at a near patient or point of care setting. ANC monitoring and early detection of CIN will not only prevent FN and subsequent compli--cations, but will also enhance capability of delivering full--dose chemotherapy on schedule for most patients. In addition, monitoring of white blood cells (WBC), specifically neutrophils, will have a number of benefits.

In the case of CIN, a doctor would typically check neutrophil count before each chemotherapy cycle. If neutrophil count is low, a patient might not receive their next cycle of chemotherapy until they have enough neutrophils. Additional neutrophil counts will enable the accurate and timely detection of a fall in neutrophils, which may indicate that the administration of growth factors may be beneficial. Patients at intermediate risk showing a drop in ANC can receive G--CSF preventing FN. This creates an additional value proposition for growth factor manufacturers. This will help to stratify cancer patients better who will benefit from G--CSF and reduce the over use of medication. Where neutrophil levels fall too low, intravenous antibiotic administration may also be advised. Monitoring and timely detection of CIN will significantly improve patient management and improve cancer treatment outcome. Therefore further research needs to be focused on identifying new and novel methods for monitoring neutrophil counts in cancer patients receiving chemotherapy.

\section{References}

1. Lyman GH, CH Lyman, O Agboola (2005) Risk models for predicting chemotherapy--induced neutropenia. Oncologist 10(6): 427-437.

2. Lyman GH, NM Kuderer (2003) Epidemiology of febrile neutropenia Support Cancer Ther 1(1): 23-35.

3. Kuderer NM, Dale DC, Crawford J, Cosler LE, Lyman GH (2006) Mortality, morbidity, and cost associated with febrile neutropenia in adult cancer patients. Cancer 106(10): 2258-2266.

4. Aapro MS, Bohlius J, Cameron DA, Dal Lago L, Donnelly JP, et al., (2011) 2010 update of EORTC guidelines for the use of granulocyte--colony stimulating factor to reduce the incidence of chemotherapy--induced febrile neutropenia in adult patients with lymphoproliferative disorders and solid tumours. Eur J Cancer 47(1): 8-32.

5. Aapro MS, Cameron DA, Pettengell R, Bohlius J, Crawford J, et al., (2006) EORTC guidelines for the use of granulocyte--colony stimulating factor to reduce the incidence of chemotherapy--induced febrile neutropenia in adult patients with lymphomas and solid tumours. Eur J Cancer 42(15): 2433-2453

6. Crawford J, DC Dale, GH Lyman (2004) Chemotherapy--induced neutropenia: risks, consequences, and new directions for its management. Cancer 100(2): 228-237.

7. Crawford J, Armitage J, Balducci L, Becker PS, Blayney DW, et al., (2013) Myeloid growth factors. J Natl Compr Canc Netw 11(10): 1266-1290.

8. Wheatley JR, Kelly WT, Tully A, Engel LA (1991) Pressure--diameter relationships of the upper airway in awake supine subjects. J Appl Physiol (1985) 70(5): 2242-2251.

9. Dunbar Angela, Tai E, Nielsen DB, Shropshire S, Richardson LC (2014) Preventing Infections During Cancer Treatment: Development of an Interactive Patient Education Website. Clin J Oncol Nurs 18.4 (2014): 426431.

10. Dale DC, McCarter GC, Crawford J, Lyman GH (2003) Myelotoxicity and dose intensity of chemotherapy: reporting practices from randomized clinical trials. J Natl Compr Canc Netw 1(3): 440-454.

11. Marshall E, H Innes (2008) Chemotherapy induced febrile neutropenia: management and prevention. Clin Med (Lond) 8(4): 448-451.

12. Dinan MA, BR Hirsch, GH Lyman (2015) Management of chemotherapyinduced neutropenia: measuring quality, cost, and value. J Natl Compr Canc Netw 13(1): e1-e7.

13. Mhaskar R, Clark OA, Lyman G, Engel Ayer Botrel T, Morganti Paladini L et al., (2014) Colony-stimulating factors for chemotherapy-induced febrile neutropenia. Cochrane Database Syst Rev (10): CD003039.

14. Mitchell S, Li X, Woods M, Garcia J, Hebard Massey K, et al., (2016) Comparative effectiveness of granulocyte colony-- stimulating factors to prevent febrile neutropenia and related complications in cancer patients in clinical practice: A systematic review. J Oncol Pharm Pract 22(5): 702716 .

15. Hoggatt J, TA Tate, LM Pelus (2015) Role of lipegfilgrastim in the management of chemotherapy-induced neutropenia. Int J Nanomedicine 10: 2647-2652.

16. Klastersky J, M Paesmans (2013) The Multinational Association for Supportive Care in Cancer (MASCC) risk index score: 10 years of use for identifying low-risk febrile neutropenic cancer patients. Support Care Cancer 21(5): 1487-1495.

17. Clark OAC, Lyman GH, Castro AA, Clark LG, Djulbegovic B (2005) Colony-Stimulating Factors for Chemotherapy--Induced Febrile Neutropenia: A Meta--Analysis of Randomized Controlled Trials. J Clin Oncol 23(18): 4198-4214.

18. Pearce A, Bradley C, Hanly P, O'Neill C, Thomas AA, et al., (2016) Projecting productivity losses for cancer--related mortality 2011-2030. BMC Cancer 16(1): 804. 
19. Lucas AJ, Olin JL, Coleman MD (2018) Management and Preventive Measures for Febrile Neutropenia. P T 43(4): 228-232.

20. Aapro Matti, Bokemeyer C, Ludwig H, Gascón P, Boccadoro M, et al., (2017) Chemotherapy-induced (febrile) neutropenia prophylaxis with biosimilar filgrastim in elderly versus non--elderly cancer patients: Patterns, outcomes, and determinants (MONITOR--GCSF study). J Geriatr Oncol 8(2): 86-95.

21. O’Brien C, Fogarty E, Walsh C, Dempsey O, Barry M, et al., (2015) The cost of the inpatient management of febrile neutropenia in cancer pa- tients-a micro-costing study in the Irish healthcare setting. Eur J Cancer Care (Engl) 24(1): 125-132.

22. Wang Li, Barron R, Baser O, Langeberg WJ, Dale DC (2014) Cancer Chemotherapy Treatment Patterns and Febrile Neutropenia in the US Veterans Health Administration. Value in Health 17(6): 739-743.

23. (2008) Systemic Anti--Cancer Therapy: For better, for worse? National Confidential Enquiry into Patient Outcome and Death. 